\section{Munc13-4, un nouvel effecteur indispensable à la sécrétion des granules cytotoxiques}

Jérôme Feldmann, Françoise Le Deist, Alain Fischer, Geneviève de Saint Basile

$>$ Les cellules cytotoxiques jouent un rôle primordial dans l'élimination des cellules tumorales ou infectées par un virus. $\varepsilon n$ quelques minutes, un lymphocyte $T$ cytotoxique qui reconnaît, par son récepteur spécifique, l'antigène viral que lui présente une cellule infectée, réorganise son cytosquelette et dirige le centre organisateur de microtubules (MTOC), l'appareil de Golgi et les granules cytotoxiques vers cette cellule cible. Les granules cytotoxiques, qui contiennent la perforine et des protéases spécialisées dans la lyse cellulaire - les granzymes - fusionnent avec la membrane plasmique du lymphocyte au niveau de la zone de contact appelée «synapse immunologique» et libèrent leur contenu dans un espace formé transitoirement entre les deux cellules (Figure 1). Ces protéines lytiques subissent alors un processus d'endocytose par la cellule cible et les granzymes sont transportés vers le noyau où ils provoquent l'apoptose de la cellule. Cette propriété, extrêmement efficace, permet à une cellule cytotoxique de tuer successivement, en quelques minutes, plusieurs cellules cibles [1].

Or cette voie effectrice de la cytotoxicité contrôle également l'homéostasie lymphocytaire chez l'homme [2, 3], et son dysfonctionnement entraîne un «syndrome hémophagocytaire» souvent fatal, caractérisé par une prolifération de lymphocytes $T$ de phénotype $\operatorname{CD} 8^{+}$, et une activation macrophagique. Ces cellules activées infiltrent les différents organes, y sécrètent de grandes quantités de cytoobservées. kines pro-inflammatoires, et entraînent leur destruction. Des images d'hémophagocytose (macrophages activés ayant phagocytés des cellules hématopoïétiques) sont

Ainsi, l'apparition d'un syndrome hémophagocytaire résulte d'une anomalie du
Inserm U.429,

Hôpital Necker-Enfants

Malades, 149, rue de Sèvres,

75015 Paris, France.

sbasile@necker.fr gène Rab27a codant pour une petite protéine $\mathrm{G}$ qui est indispensable à l'exocytose des granules cytotoxiques chez les patients atteints du syndrome de Griscelli du type 2 [4]. Une anomalie du gène CHS/LYST, qui induit une augmentation de la taille et un défaut d'excrétion des granules cytotoxiques, peut provoquer également un syndrome hémophagocytaire chez les patients présentant un syndrome de Chédiak-Higashi $[5,6]$. Rab27a et CHS/LYST sont également nécessaires au transfert au niveau de l'épiderme, du pigment des mélanocytes vers les kératinocytes adjacents,

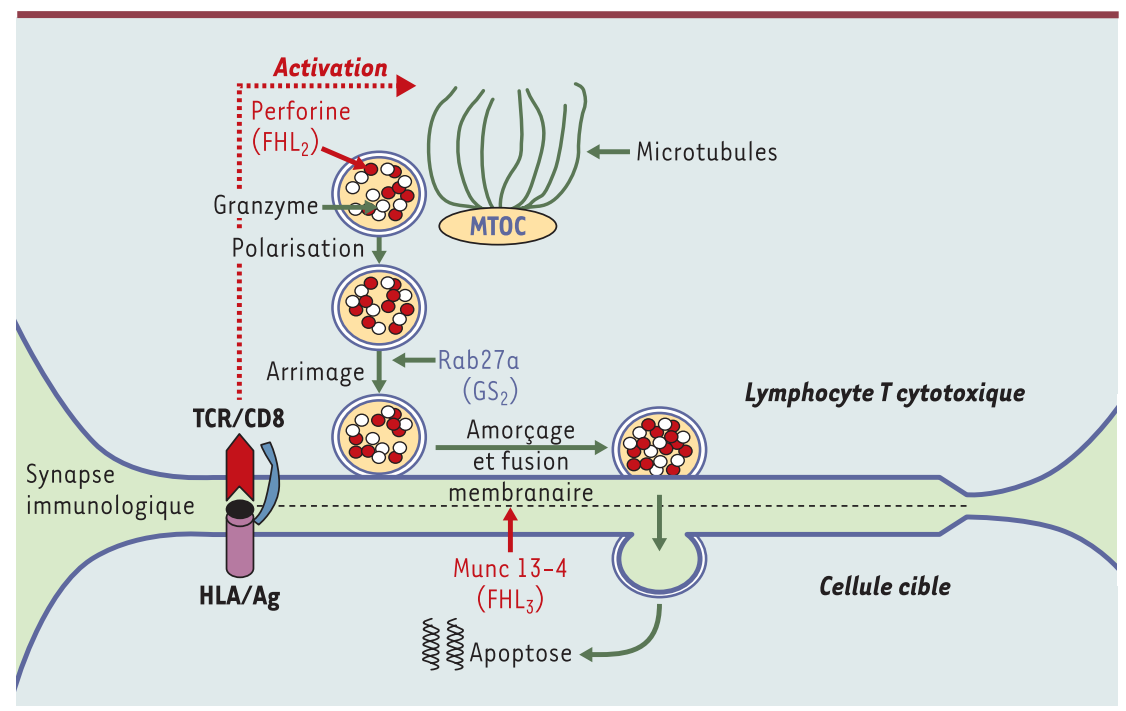

Figure 1. Schéma illustrant l'étape de la voie d'excrétion des granules cytotoxiques, ciblée par Munc13-4. Un lymphocyte T CD8 cytotoxique qui reconnaît par son récepteur T (TCR) l'antigène présenté par la molécule HLA (HLA/Ag) d'une cellule cible, forme transitoirement une synapse immunologique avec cette cellule. Après ce contact cellulaire, les granules cytotoxiques qui contiennent la perforine et les granzymes se dirigent le long des microtubules vers la cellule présentatrice d'antigène. Ils atteignent la membrane plasmique du lymphocyte et s'y arriment après une dernière étape de transport qui requiert Rab27a. Munc13-4 est ensuite nécessaire pour conférer aux granules cytotoxiques la capacité de fusionner avec la membrane plasmique, permettant ainsi la libération de leur contenu dans la fente synaptique. Ce contenu sera endocyté par la cellule cible et entraînera sa lyse. Un défaut en perforine conduit à la lymphohistiocytose de type 2 (FHL2), un défaut en Rab27a, au syndrome de Griscelli de type 2 (GS2) et un défaut en Munc13-4 à la lymphohistiocytose de type 3 (FHL3). MTOC: centre organisateur des microtubules. 
expliquant l'albinisme partiel qui accompagne ces maladies

$(\rightarrow)$. L'apparition d'un syndrome hémophagocytaire est la seule expres-

$(\rightarrow) \mathrm{m} / \mathrm{s}$ 2000, n० 6-7, p. 745 sion phénotypique d'une troisième affection héréditaire de transmission autosomique récessive, connue sous le nom de lymphohistiocytose familiale (LHF), dans laquelle nous avons montré précédemment que $30 \%$ des patients (groupe FHL2) présentent des mutations du gène de la perforine $[7,8]$.

Implication d'un nouvel effecteur, hMunc13-4, dans

la lymphohistiocytose familiale

L'analyse exhaustive de l'activité cytotoxique des lymphocytes de tous les patients présentant une LHF, et chez lesquels une anomalie du gène de la perforine avait été exclue, nous a permis d'identifier un sous-groupe de patients (FHL3), présentant un défaut d'activité cytotoxique sévère des lymphocytes $T$ et des cellules de l'immunité innée (natural killer), indépendant de la perforine. Une forte liaison génétique entre la ségrégation de la maladie dans ces familles et une région génétique en 17 q25 a été mise en évidence. La recherche de gènes candidats nouvellement identifiés dans les bases de données du génome humain a permis de repérer dans cette région la présence de l'orthologue humain du gène Munc13-4 du rat. Six anomalies différentes de ce gène ont été identifiées chez ces patients, qui toutes entraînent une altération de la protéine hMuncl3-4, principalement des délétions de la partie carboxy-terminale de la protéine. La restauration de l'activité cytotoxique des lymphocytes de patients dans lesquels l'expression d'une forme fonctionnelle de hMunc13-4 avait été induite prouvait la relation de cause à effet entre l'altération de la protéine et le phénotype clinique [9].

\section{Expression et fonction de hMunc13-4}

Aucune fonction de Munc13-4 n'avait été identifiée chez le rat. La similitude d'ar- chitecture des domaines de Munc13-4 et de ceux des protéines de la famille Muncl3/Uncl3 explique que Muncl3-4 a été inclus dans cette famille [10]. Les protéines Muncl3 sont exclusivement ou très majoritairement exprimées dans le cerveau, et un rôle important de ces protéines dans la sécrétion du contenu des vésicules synaptiques neuronales a été postulé [11, 12]. Or hMunc13-4 n'est pas exprimé dans le cerveau mais de façon prédominante dans les cellules hématopoïétiques ainsi que dans d'autres tissus, comme l'épithélium pulmonaire et le placenta.

Nos études nous ont permis de démontrer que le mécanisme d'excrétion des granules cytotoxiques au niveau de la synapse immunologique est comparable à celui des vésicules synaptiques au niveau de la synapse neurologique. HMunc13-4 est un effecteur indispensable à la sécrétion «contrôlée» des protéines lytiques stockées dans des granules préformés, mais elle n'intervient pas dans la voie de sécrétion «constitutive» des cytokines (dont l'interféron $\gamma$ ) utilisée par les lymphocytes. HMunc13-4 agit à une étape tardive de la voie de sécrétion, puisque son absence dans les lymphocytes de patients n'affecte ni la polarisation du MTOC, de l'appareil de Golgi ou des granules cytotoxiques, ni l'arrimage des granules lytiques à la membrane plasmique au niveau de la synapse immunologique. HMuncl3-4 agit donc en aval de Rab27a qui est nécessaire à cette dernière étape du transport des granules polarisés vers la membrane plasmique (Figure 1). En utilisant une forme fluorescente de hMuncl3-4 nous avons montré que cette protéine polarise avec les granules cytotoxiques et se localise au niveau de la synapse immunologique, et qu'en son absence, aucune image de fusion ou d'excrétion des granules cytotoxiques au niveau de la synapse immunologique n'est observée. HMuncl3-4 confère aux vésicules un état de «compétence» indispensable pour amorcer l'étape de fusion membranaire qui pré- cède l'excrétion du contenu des granules. Il y a donc une grande similitude des mécanismes d'excrétion des granules cytotoxiques au niveau de la synapse immunologique et des vésicules synaptiques au niveau de la synapse neurologique. Dans ces deux situations, il existe une étape décisive entre l'association des vésicules à la membrane plasmique et la fusion membranaire, sous le contrôle des isoformes de Muncl3 (Munc13-1,2,3 dans le cerveau, Muncl34 dans les lymphocytes). Au niveau de la synapse neurologique, les isoformes de Muncl3 contrôlent également le nombre de vésicules qui acquièrent cette compétence parmi celles qui sont pré-localisées au niveau de la membrane [14], participant ainsi à la plasticité synaptique. Dans les lymphocytes, la localisation des granules cytotoxiques à la membrane plasmique n'a lieu qu'après la formation de la synapse immunologique [1]. II reste à déterminer si Muncl3-4 exerce également un rôle dans la sélection d'un quantum de granules excrétés à chaque contact du lymphocyte avec une cellule cible, conférant aux lymphocytes cytotoxiques cette fonction de «tueur en série».

On peut s'étonner qu'aucune différence phénotypique n'existe entre les patients présentant des anomalies génétiques de hMunc13-4 ou de la perforine, l'expression de hMuncl3-4 n'étant pas restreinte aux cellules hématopoïétiques, contrairement à la seconde. II est possible que les conséquences du défaut d'excrétion dans les autres tissus ne soient pas suffisamment sévères pour s'exprimer, ou qu'elles y soient masquées par un mécanisme redondant d'excrétion «contrôlée» des vésicules.

Munc13-4 est le quatrième effecteur (mais peut-être pas le dernier) identifié dans la voie de sécrétion des granules cytotoxiques, dont l'anomalie conduit au dérèglement de la réponse lymphocytaire TCD $8^{+}$telle qu'on l'observe au cours du syndrome hémophagocytaire. Ces travaux illustrent à nouveau la puissance de l'étude systématique d'un ensemble 
de maladies héréditaires dans la caractérisation de grandes voies physiologiques. D'un point de vue médical, la caractérisation moléculaire de cette nouvelle forme de LHF permettra de mieux distinguer, face à un cas familial unique, les formes héréditaires des formes acquises de syndrome hémophagocytaire. Le conseil génétique et l'attitude thérapeutique pourront être ainsi adaptés. $\bullet$

Munc13-4 is essential

for cytolytic granule fusion

\section{REMERCIEMENTS}

Nous tenons à remercier l'Inserm, l'Association Française contre le Cancer, La Fondation pour la recherche Médicale et Vaincre les Maladies Lysosomales qui soutiennent généreusement nos travaux.

\section{RÉFÉRENCES}

1. Stinchcombe JC, Bossi G, Booth S, Griffiths GM.

The immunological synapse of CTL contains a secretory domain and membrane bridges. Immunity 2001 ; 15: 751-61.

2. De Saint Basile G. Implication du trafic intracellulaire dans trois maladies héréditaiores du système hématopoïétique. Med Sci (Paris) $2000 ; 16$ : 745-50.

3. de Saint Basile G, Fischer A. The role of cytotoxicity in lymphocyte homeostasis. Curr Opin Immunol 2001 ; $13: 549-54$.

4. Ménasché G, Pastural \& , Feldmann J, et al. Mutations in RAB27A cause Griscelli syndrome associated with hemophagocytic syndrome. Nat Genet 2000 ; $25: 173-6$.
5. Nagle DL, Karim AM,

Woolf $\varepsilon A$, et al.

Identification

and mutation analysis

of the complete gene for

Chediak-Higashi syndrome.

Nat Genet 1996 ;

$14: 307-11$.

6. Barbosa MDFS, Nguyen $Q A$, Tchernev VT, et al.

Identification

of the homologous beige

and Chediak-Higashi syndrome genes. Nature 1996 ; 382 : 262-5.

7. Dufourcq Lagelouse R, Le Deist F, Fischer A, de Saint Basile G. Altération du gène codant pour la perforine dans la lymphohistiocytose familiale. Med Sci (Paris) 1999 ; 15 : 1479-81.

8. Stepp S, DufourcqLagelouse R, Le Deist F, et al. Perforin Gene Defects in Familial Hemophagocytic Lymphohistiocytosis. Science 1999 ; $286: 1957-9$.
9. Feldmann J, Callebaut I, Raposo G, et al. Munc13-4 is essential for cytolytic granules fusion and is mutated in a form of familial hemophagocytic lymphohistiocytosis (FHL3). Cell 2003; 115 : 461-73.

10. Koch H, Hofmann K, Brose N. Definition of Munc13-homologydomains and characterization of a novel ubiquitously expressed Muncl3 isoform. Biochem J 2000 ; 349: 247-53.

11. Betz A, Thakur P, Junge HJ, et al. Functional interaction of the active zone proteins Muncl3-1 and RIMl in synaptic vesicle priming. Neuron 2001; 30: 183-96.

12. Rizo J, Sudhof TC. Snares and Munc18 in synaptic vesicle fusion. Nat Rev Neurosci 2002 ; 3 : 641-53.

13. Rosenmund C, Sigler A, Augustin I, Reim K, Brose N, Rhee JS. Differential control of vesicle priming and short-term plasticity by Muncl3 isoforms. Neuron $2002 ; 33: 411-24$.

\section{NOUVELLE}

\section{Du rythme dans le foie en régénération}

Michèle Teboul, Béatrice Rayet, Franck Delaunay
Université

de Nice-Sophia Antipolis, CNRS UMR6078,

284, chemin du Lazaret, 06230 Villefranche-sur-Mer, France.

teboulm@unice.fr

cycle cellulaire ont été décrites dans l'épithélium intestinal et la peau [4]. L'équipe d'Hitoshi Okamura à l'Université de Kobe (Japon) vient d'apporter un élément décisif en faveur de ce lien en démontrant que, lors de la régénération hépatique, l'horloge circadienne contrôle l'expression de gènes du cycle cellulaire et finalement la mitose [5]. 